\title{
First Occurrence Prime Gaps
}

\author{
By Jeff Young and Aaron Potler
}

\begin{abstract}
An ongoing search for first occurrence prime gaps continues.
\end{abstract}
An ongoing search for first occurrence prime gaps is being carried out which extends all previous work done on this subject. To date this search has found all such gaps for primes up to $7.263 \times 10^{13}$. First occurrence prime gaps had previously been known for primes less than $4.444 \times 10^{12}$ [2]. Several gaps larger than the previously largest gap of 682 (not a first occurrence) found by Weintraub [4] have been found.

Computer programs were written in FORTRAN and CAL (Cray Assembly Language) on a CRAY-2 supercomputer.

The computation was conducted as follows. A sufficient number of primes were generated to perform a sieve. Odd numbers beginning with 3 were sieved one block at a time, where each block was chosen to contain 40,000,000 numbers based on system resource availability. The even numbers were eliminated during initialization of each block. One number was stored per 64-bit computer word. After sieving each block, the differences between consecutive primes were calculated and stored. This was accomplished by loading 64 elements of the sieved block at a time into a vector register. A 64-bit vector mask was created containing 1's for corresponding nonzero values in the vector register. If the vector mask was zero, the next 64 values of the sieved block were loaded into the vector register. If the mask was nonzero, an instruction to count the number of leading zeros was executed to get the offset from the beginning of the vector register for the next prime. A subtraction of the previous prime was done, thus arriving at the gap. The leftmost 1 of the vector mask was cleared and the method repeated, beginning with checking if the vector mask was zero. This was repeated until the entire block was processed. The last prime in each block was saved in order to calculate the difference between that prime and the first prime in the next block to make sure no gaps were missed. The time to perform the sieve for each block, and to calculate all gaps generated, was about 10.5 seconds for numbers in the range of $7.2 \times 10^{13}$. The largest prime in the last block processed to date is 72635119999997 , so the table of first occurrence prime gaps is complete to that prime.

The following table lists all the first occurrence prime gaps found. This table agrees with all previously published results [2], [3], [1]. The maximal first occurrence prime gaps are marked with an asterisk (*).

Received June 8, 1987; revised November 16, 1987.

1980 Mathematics Subject Classification (1985 Revision). Primary 11A41. 
following gap the prime following gap the prime following

gap the prime
* 2

* 4

* 6

* 8

10

12

* 14

16

* 18

* 20

* 22

24

26

28

30

32

* 34

* 36

38

40

42

* 44

46

48

50

* 52

54

56

58

60

62

64

66

68

70

* 72

74

76

78

80

82

84

* 86

88

90

92

94

* 96

98

100

$3 \quad 102$

1129

1669

2477

2971

4297

5591

1327

9551

30593

19333

16141

15683

81463

28229

31907

19609

35617

82073

44293

43331

34061

89689

162143

134513

173359

31397

404597

212701

188029

542603

265621

461717

155921

544279

404851

927869

1100977

360653

604073

396733
104

106

108

110

* 112

* 114

116

* 118

120

122

1444309

1388483

1098847

2238823

1468277

370261

492113

5845193

1349533

1895359

3117299

6752623

1671781

3851459

5518687

1357201

6958667

6371401

3826019

7621259

10343761

11981443

6034247

2010733

13626257

8421251

4652353

17983717

49269581

33803689

39175217

$\begin{array}{ll}164 & 20285099 \\ 166 & 83751121\end{array}$

$168 \quad 37305713$

$170 \quad 27915737$

$172 \quad 38394127$

$174 \quad 52721113$

$176 \quad 38089277$

$178 \quad 39389989$

- 180

17051707

$182 \quad 36271601$

$184 \quad 79167733$

$186 \quad 147684137$

$\begin{array}{ll}188 & 134065829\end{array}$

$190 \quad 142414669$

$192 \quad 123454691$

$194 \quad 166726367$

$196 \quad 70396393$

$198 \quad 46006769$

$200 \quad 378043979$
$202 \quad 107534587$

$204 \quad 112098817$

$206 \quad 232423823$

$208 \quad 192983851$

* $210 \quad 20831323$

$212 \quad 215949407$

$214 \quad 253878403$

$216 \quad 202551667$

$218 \quad 327966101$

* $220 \quad 47326693$

* $222 \quad 122164747$

$224 \quad 409866323$

$226 \quad 519653371$

$228 \quad 895858039$

$230 \quad 607010093$

$232 \quad 525436489$

* $234 \quad 189695659$

$236 \quad 216668603$

$238 \quad 673919143$

$240 \quad 391995431$

$242 \quad 367876529$

$244 \quad 693103639$

$246 \quad 555142061$

* $248 \quad 191912783$

* 250387096133

$252 \quad 630045137$

$254 \quad 1202442089$

$\begin{array}{ll}256 & 1872851947\end{array}$

$258 \quad 1316355323$

$260 \quad 944192807$

$\begin{array}{ll}262 & 1649328997\end{array}$

$264 \quad 2357881993$

$\begin{array}{ll}266 & 1438779821\end{array}$

$\begin{array}{ll}268 & 1579306789\end{array}$

$270 \quad 1391048047$

$272 \quad 1851255191$

$274 \quad 1282463269$

$276 \quad 649580171$

$278 \quad 4260928601$

$280 \quad 1855047163$

* $282 \quad 436273009$

$284 \quad 1667186459$

$286 \quad 2842739311$

* 2881294268491

$290 \quad 1948819133$

* 2921453168141

$294 \quad 5692630189$

$296 \quad 5260030511$

$298 \quad 8650524583$

$300 \quad 4758958741$ 


\begin{tabular}{|c|c|c|c|c|c|}
\hline gap & $\begin{array}{l}\text { following } \\
\text { the prime }\end{array}$ & gap & $\begin{array}{l}\text { following } \\
\text { the prime }\end{array}$ & gap & $\begin{array}{l}\text { following } \\
\text { the prime }\end{array}$ \\
\hline 302 & 6675573497 & 402 & 44293346177 & 502 & 1258535916601 \\
\hline 304 & 2433630109 & 404 & 144999022043 & 504 & 747431049203 \\
\hline 306 & 3917587237 & 406 & 49306638307 & 506 & 1339347750707 \\
\hline 308 & 5490459101 & 408 & 134664608389 & 508 & 1841086484491 \\
\hline 310 & 4024713661 & 410 & 98276144093 & 510 & 2209016910131 \\
\hline 312 & 6570018347 & 412 & 124221464119 & 512 & 1999066711391 \\
\hline 314 & 8948418749 & 414 & 49914935177 & * 514 & 304599508537 \\
\hline 316 & 12109172293 & 416 & 1219721 & * 516 & 416608695821 \\
\hline 318 & 4372999721 & 418 & 129300694603 & 518 & 2296497058133 \\
\hline 320 & 2300942549 & 420 & 82490815123 & 520 & 2336167262449 \\
\hline 322 & 7961074441 & 422 & 280974865361 & 522 & 1214820695701 \\
\hline 324 & 10958687879 & 424 & 264495345259 & 524 & 2256065636039 \\
\hline 326 & 58379 & 426 & 180265 & 526 & 162050 \\
\hline 328 & 130868 & 428 & 219950 & 528 & 152974 \\
\hline 330 & 629135 & 430 & 25096419 & 530 & 220549237 \\
\hline 332 & 5893180121 & 432 & 87241 & * 53 & 461690510011 \\
\hline 334 & 30827138509 & 434 & 127084 & * 534 & 614487453523 \\
\hline 336 & 3842610773 & 436 & 367459 & 536 & 5371284217763 \\
\hline 338 & 220763 & 438 & 10132 & 5 & 212253 \\
\hline 340 & 86052 & 440 & 14184 & * 540 & 73883 \\
\hline 342 & 120107 & 442 & 417470 & 542 & 2707053887651 \\
\hline 344 & 197240 & 444 & 36172 & 544 & 2652427555639 \\
\hline 346 & 1129140 & 446 & 1904180 & 546 & 2164206784721 \\
\hline 348 & 17002876643 & 448 & 402872474743 & 548 & 3380058341279 \\
\hline 350 & 168087 & 450 & & 550 & 2496646209271 \\
\hline 352 & 307508 & 452 & 4668 & & 221 \\
\hline 354 & 43024 & 454 & 202530 & 5 & 36211 \\
\hline 356 & 243550 & * 456 & 25056082087 & 556 & 4338624362173 \\
\hline 358 & 1679232 & 458 & 304040 & 558 & 5263973982823 \\
\hline 360 & 20068818197 & 460 & 1319562 & 560 & 4260199366373 \\
\hline 362 & 358777 & 462 & 400729 & 562 & 2081209441279 \\
\hline 364 & 2542 & 464 & & & 1480 \\
\hline 366 & 97 & 466 & 565855 & 566 & 489 \\
\hline 368 & 51430518413 & * 468 & 127976334671 & 568 & 6010330572331 \\
\hline 370 & 59942358571 & 470 & 681753256133 & 570 & 4442109925217 \\
\hline 372 & 20404 & 472 & 865244 & & 5441175346967 \\
\hline 374 & 2306 & * 474 & 18222 & 574 & 31087 \\
\hline 376 & 161616 & 476 & $725978 \mathrm{~s}$ & 576 & 8817792098461 \\
\hline 378 & 381169 & 478 & 367766 & 578 & 7552870120721 \\
\hline 380 & 23323808741 & 480 & 482423533897 & 580 & 9383081340541 \\
\hline 382 & 10726904659 & 482 & 10516027 & * 582 & 1346294310749 \\
\hline 384 & 206780 & 484 & & & 6993007248239 \\
\hline 386 & 352386 & * 486 & 43 & 586 & 6364466316577 \\
\hline 388 & 156798792223 & 488 & 1275363152099 & * 588 & 1408695493609 \\
\hline 390 & 53241805651 & * 490 & 297501075799 & 590 & 20761252261751 \\
\hline 392 & 117215204531 & 492 & 910361180689 & 592 & 3410069454097 \\
\hline 394 & 22367084959 & 494 & 804541404419 & 594 & 5499789519863 \\
\hline 050 & 50806025873 & 49 & 880318998907 & 596 & 10487995154603 \\
\hline 398 & & 498 & 428315806823 & 598 & 5614481773561 \\
\hline 400 & 47203303159 & * 500 & 303371455241 & 600 & 4872634110067 \\
\hline
\end{tabular}




\begin{tabular}{|c|c|c|c|c|c|}
\hline gap & $\begin{array}{l}\text { following } \\
\text { the prime }\end{array}$ & gap & $\begin{array}{l}\text { following } \\
\text { the prime }\end{array}$ & gap & $\begin{array}{l}\text { following } \\
\text { the prime }\end{array}$ \\
\hline 602 & 1968188556461 & 642 & 14141685364577 & 690 & 15712145060693 \\
\hline 604 & 5439564948583 & 644 & 41433781612373 & 692 & 43603583701331 \\
\hline 606 & 12112937821403 & 646 & 51027160468351 & 694 & 62088893223739 \\
\hline 608 & 20767330530329 & 648 & 9787731507761 & 696 & 23333096984797 \\
\hline 610 & 9105981382177 & 650 & 5120731250207 & 698 & 33785727371453 \\
\hline 612 & 13397310636587 & * 652 & & 700 & 14998144209049 \\
\hline 614 & 17418 & 654 & 549 & 702 & \\
\hline 616 & 80952245 & 656 & 658 & 706 & 3562575 \\
\hline 618 & 416563339 & 658 & 39883132 & 714 & 4963999 \\
\hline 620 & 93440930 & 660 & 106 & 716 & 13829048559701 \\
\hline 622 & 1305996994 & 662 & 11082394066097 & 724 & 59692452 \\
\hline 624 & 24923033918059 & 664 & 38745678640849 & 728 & 57723522921803 \\
\hline 626 & 33605 & 666 & & 730 & 24179270 \\
\hline 628 & 34140 & 668 & & 740 & 57360609 \\
\hline 630 & 126444611 & 670 & 4713 & 744 & 4261 \\
\hline 632 & 456786858 & 672 & 26456514 & 756 & 70099348325843 \\
\hline 634 & 17659394869309 & * 674 & 7177162611713 & 758 & 47581758352253 \\
\hline 636 & 9483480841753 & 678 & 37970994487033 & * 766 & 19581334192423 \\
\hline 638 & 17499522060011 & 682 & 41459443375351 & * 778 & 42842283925351 \\
\hline 640 & 22099408494481 & 684 & 30236507704253 & & \\
\hline
\end{tabular}

Cray Research, Inc.

1440 Northland Drive

Mendota Heights, Minnesota 55120

1. R. BRENT, "The first occurrence of large gaps between successsive primes," Math. Comp., v. 27,1973, pp. 959-963.

2. R. BRENT, "The first occurrence of certain large prime gaps," Math. Comp., v. 35, 1980, pp. 1435-1436.

3. L. J. LANDER \& T. R. PARKIN, "On first appearance of prime differences," Math. Comp., v. 21,1967 , pp. $483-488$.

4. S. WeintraUB, "A prime gap of 682 and a prime arithmetic sequence," BIT, v. 22, 1982, p. 538 . 